\title{
Implications of a defined daily dose fixed database for drug utilization research studies: The case of statins in Portugal
}

\author{
Catarina Abrantes $^{1}$ | Fernanda S. Tonin ${ }^{2}$ (ㅇ | Joana Reis-Pardal ${ }^{3}$ (i) | \\ Margarida Castel-Branco ${ }^{1}$ () | Claudia Furtado ${ }^{4}$ | Isabel V. Figueiredo ${ }^{1}$ (๑) | \\ Fernando Fernandez-Llimos ${ }^{5}$
}

${ }^{1}$ Pharmacology and Pharmaceutical Care Laboratory, Faculty of Pharmacy, University of Coimbra, Coimbra, Portugal

${ }^{2}$ Pharmaceutical Sciences Postgraduate Program, Federal University of Paraná, Curitiba, Brazil

${ }^{3}$ Pharmacy Department, Hospital de Sant'Ana, Parede, Portugal

${ }^{4}$ Health Technology Assessment Department, National Authority of Medicines and Health Products (INFARMED), Lisbon, Portugal

${ }^{5}$ Laboratory of Pharmacology, Department of Drug Sciences, Faculty of Pharmacy,

University of Porto, Porto, Portugal

\section{Correspondence}

Fernando Fernandez-Llimos; Laboratory of Pharmacology, Department of Drug Sciences, College of Pharmacy, University of Porto, R. Jorge de Viterbo Ferreira 228, 4050-313

Porto, Portugal.

Email: fllimos@ff.up.pt
Aims: Given the discrepancies between PDDs (prescribed daily doses) and DDDs (defined daily doses), we aimed to assess the extent of error in the results of an 18-year population-level study on statin utilization in Portugal.

Methods: The Portuguese regulatory agency provided data for the period 2000-2018 on statin dispensing (C10AA). The DDDs were gathered from the ATC/DDD database. DDDs were calculated by the DDD year-by-year approach (DDD ${ }_{\text {YEAR }}$ ) and by the DDD last-year approach (DDD according to the year-by-year approach (PDD YEAR $)$. Statin annual utilization rates per 1000 inhabitants per day were also calculated. Percent errors were calculated for PDD YEAR and DDD YEAR units.

Results: The DDD YEAR approach revealed decreases in the consumption of atorvastatin, fluvastatin, lovastatin, pravastatin and simvastatin in 2009, when their DDD was modified. Conversely, the results from both DDD LAST $_{\text {and PDD }}$ YEAR approaches indicated gradual changes in the actual consumption of all statins in Portugal. Before 2009 , atorvastatin, pravastatin and simvastatin utilization was greatly overestimated by DDD ${ }_{\text {YEAR }} / 1000$ inhabitants/day. The average dose of lovastatin prescribed in the past 18 years $(20 \mathrm{mg}$ ) was below the assigned DDDs during the study period, varying from $30 \mathrm{mg}$ to $45 \mathrm{mg}$. Conversely, the PDD for fluvastatin was above the DDD values (ranging from $40 \mathrm{mg}$ in 2000 to $70 \mathrm{mg}$ in 2016). For atorvastatin, pravastatin and simvastatin, national PDDs were above the assigned DDD until the DDD modification in 2009.

Conclusions: A more dynamic system, based on national and annually updated DDDs, should be able to reduce discrepancies between DDDs and PDDs and the bias in utilization studies.

KEYWORDS

defined daily dose, drug utilization, reproducibility of results 


\section{1 | INTRODUCTION}

The defined daily dose (DDD) system was developed by the World Health Organization (WHO) Drug Utilization Research Group as an international tool to standardize drug consumption data from different sources. ${ }^{1}$ The DDD has become the gold standard unit of measurement for drug utilization research (DUR) studies ${ }^{2-9}$ and is defined as "the assumed average maintenance dose per day for a drug used for its main indication in adults". ${ }^{10}$

The DDD of a drug is periodically reviewed by the WHO Collaborating Centre for Drug Statistics Methodology (WHO-CC-DMS), and its value can change over time. ${ }^{10}$ The first revision of a DDD is undertaken 3 years after its assignment. After that, DDDs usually remain unchanged for at least 5 years. Although the WHO accepts the update of DDDs, "changes of DDDs should be kept to a minimum and avoided as far as possible", aiming at maintaining a fixed database for DUR studies. ${ }^{10}$

The prescribed daily dose (PDD) is defined as the average dose prescribed according to a representative sample of prescriptions. ${ }^{11}$ The PDD of a drug is commonly set from patient-level prescription studies and can geographically differ depending on several factors, such as therapeutic indication, illness severity, sex, age, pharmacogenetics characteristics, and whether a drug is used as mono- or combined therapy. ${ }^{12}$ Studies demonstrated that for many drugs, maximum doses used in countries like Japan were much lower than in the United States. Additionally, doses approved by regulatory bodies often vary between countries, ${ }^{13-15}$ which contributes to discrepancies across national PDDs.

When a good agreement between PDD and DDD exists, drug consumption figures, expressed as number of DDDs/1000 inhabitants/day, provide an estimate of the fraction of drug users within a population. ${ }^{11}$ For instance, a value of 10 DDD/1000 inhabitants/day indicates that, on average, $1 \%$ of the population (10 in 1000) is treated daily with a specific drug. ${ }^{12}$

However, several studies have demonstrated discrepancies between DDDs and PDDs. ${ }^{16-20}$ Walley et al. ${ }^{17}$ found differences in the PDD of statins among European Union member states, with more than a two-fold range in variance. To better understand the bias introduced in DUR studies by these figures, an analysis of statin utilization can be valuable, especially due to the high concordance rate between the dispensed tablet strength and the PDDs of these drugs. ${ }^{21}$ Statins are a perfect group to study the accuracy of DDD assignment because, similar to the administration of other drugs (e.g., bisphosphonates), statin use follows a homogeneous administration pattern (once a day), but different from bisphosphonates, statins are prescribed at different doses according to patients' needs. As such, statin consumption figures based on PDDs can be easily determined from aggregate dispensing data without the need to access patient-level information.

Therefore, we aimed to investigate the extent of error in the results of a national 18-year population-level study on statin utilization due to discrepancies between PDDs and DDDs to provide recommendations to improve the DDD system.

\section{What is already known about this subject}

- The World Health Organization created the defined daily dose system to be the standards metric of drug utilization studies worldwide.

- The World Health Organization maintains a global DDD database and avoids DDD changes as far as possible.

- DDD per 1000 inhabitants and day should represent the proportion of people consuming the drug per 1000 population.

\section{What this study adds}

- Differences in national prescribing patterns substantially influence the reliability of DDD/1000 inhabitants/day as the drug utilization metric.

- Periodically modified and nationally adapted DDDs would increase the DDD system reliability as the drug utilization metric.

\section{2 | METHODS}

\subsection{Data sources}

Data on statin dispensing (drugs from the ATC group: C10AA - HMG CoA reductase inhibitors, including atorvastatin, cerivastatin, fluvastatin, lovastatin, pitavastatin, pravastatin, rosuvastatin, simvastatin) were obtained from the Portuguese National Authority of Medicines and Health Products (INFARMED). Data represented the total number of statin packages dispensed to ambulatory patients and reimbursed by the Portuguese National Health Service from 1 January 2000 to 31 December 2018. For each package, the strength (dose per administration unit) and the number of units (i.e., tablets or capsules) were available. National population data were obtained from the Population Estimative at PORDATA database (Foundation Francisco Manuel dos Santos, Lisbon), available at https://www. pordata.pt/.

The DDDs assigned to each drug were gathered from the ATC/DDD database provided by the WHO Collaborating Center for Drug Statistics Methodology (https://www.whocc.no/atc_ddd_index/), with additional consultation of the list of DDD alterations from 2005 to 2020 (https://www.whocc.no/atc_ddd_alterations_cumulative/ddd_ alterations/). Alterations to the DDDs of some statins occurred during the study period. In 2009, the DDDs of atorvastatin, fluvastatin, lovastatin, pravastatin and simvastatin changed from 10 to 20,40 to 60,30 to 45,20 to 30 and 15 to 30 , respectively. 


\subsection{Statin utilization metrics}

The DDDs were calculated by dividing the total number of milligrams consumed in a given time period by the DDD assigned by the WHO in milligrams using the following equation:

$\mathrm{DDD}=\frac{\text { Tablets in } \mathrm{mg}_{\text {per }_{\text {package }}} \times \text { Number of Tablets }_{\text {per }_{\text {package }}} \times \text { Number }_{\text {packages }}}{\operatorname{DDD}_{\mathrm{WHO}}(\mathrm{mg})}$

Two different approaches were used for this calculation: (1) the DDD year-by-year approach, ${ }^{22}$ in which drug utilization was calculated using the DDD unit existing in the year under analysis (DDDYEAR); and (2) the DDD last-year approach, ${ }^{22}$ in which drug utilization was calculated each year using the DDD unit existing at the end of the study period (DDD LAST $_{\text {). }}$.

Alternatively, based on the assumption that the statin prescribed dose is equal to the strength of one tablet (i.e., assuming that all patients took one statin tablet daily), ${ }^{23,24}$ the consumed PDD was calculated by multiplying the number of tablets per package by the number of dispended packages in a given period:

PDD $=$ Number of Tablets $_{\text {per }_{\text {package }}} \times$ Number $_{\text {packages }}$

The results were reported according to the PDD year-by-year approach, in which drug utilization is calculated each year using the national PDD of that year (PDD YEAR).

Finally, following international recommendations, ${ }^{12}$ we calculated statin annual utilization rates per 1000 inhabitants per day. As such, three rates were calculated based on the three previously mentioned measurement units: (1) the DDD $D_{\text {YEAR }} / 1000$ inhabitants/day; (2) the $\mathrm{DDD}_{\text {LAST }} / 1000$ inhabitants/day; and (3) the $\mathrm{PDD}_{\mathrm{YEAR}} / 1000$ inhabitants/day.

\section{3 | Percent error calculation}

Percent errors were calculated for both DDD (i.e., PDD YEAR and DDD $D_{\text {YEAR }}$ ) units using the following equation:

$$
\text { Error }_{\%}=\frac{(\mathrm{DDD}[\mathrm{x}]-\mathrm{PDD})}{\mathrm{PDD}} \times 100
$$

Percent errors with a positive sign represent an overestimation of the proportion of users by the DDD system, while percent errors with a negative sign represent an underestimation of users by the DDD system.

\subsection{Nomenclature of targets and ligands}

Key protein targets and ligands in this article are hyperlinked to corresponding entries in http://www.guidetopharmacology.org, the common portal for data from the IUPHAR/BPS Guide to
PHARMACOLOGY, and are permanently archived in the Concise Guide to PHARMACOLOGY 2019/20.

\section{3 | RESULTS}

Dispensing data for the entire time period of the study (2000-2018) were available for atorvastatin, fluvastatin, lovastatin, pravastatin and simvastatin. Cerivastatin was withdrawn from the market for safety reasons in August 2001. Rosuvastatin was first marketed in 2004 and pitavastatin in 2011.

Considerable differences were observed in the utilization rates of several statins depending on the approach used to calculate them (Figure 1). The application of the DDD YEAR approach revealed a sharp theoretical decrease in the consumption of atorvastatin, fluvastatin, lovastatin, pravastatin and simvastatin in 2009 (blue line), when their DDD was modified. This event was highly pronounced in the case of simvastatin, where a theoretical decrease of approximately $40 \%$ was observed in 1 year. Conversely, the results from both the DDD LAST (red line) and the PDDYEAR (green line) approaches indicated that gradual changes occurred in the actual consumption of all statins. The three approaches indicate a similar temporal evolution of rosuvastatin, cerivastatin and pitavastatin utilization, as demonstrated by the perfect overlapping of all three utilization curves.

It is clear that DDD YEAR $/ 1000$ inhabitants/day revealed almost no error compared to DDD $D_{\text {LAST }} / 1000$ inhabitants/day for cerivastatin, pitavastatin and rosuvastatin during the study period. For all the remaining statins, DDD absolute percent errors in almost all years, with both lovastatin and fluvastatin reaching values over $50 \%$ and $80 \%$, respectively (Figure 2). Before 2009, the utilization of atorvastatin, pravastatin and simvastatin was greatly overestimated by DDD $D_{\text {YEAR }} / 1000$ inhabitants/day, whereas after that year, the opposite was observed. For instance, in 2007, 23691808 atorvastatin doses were prescribed accounting for 32666396 DDDs, while the 30643082 doses prescribed in 2010 corresponded to 21176292 DDDs. Similarly, the 23752810 pravastatin doses prescribed in 2007 were equal to 31166472 DDDs, while these values were 31427390 and 20313213 , respectively for 2015. Or the 283769972 simvastatin doses prescribed in 2008 corresponded to 393025568 DDDs, but the 412419539 doses in 2010 were only 293207120 DDDs. Lovastatin utilization was always underestimated, initially by slightly more than 30\% (10 997030 doses vs. 7383903 DDDs in 2000) and, from 2009 onward, by slightly more than 50\% (2420 320 doses vs. 1124204 DDDs in 2018). Figures for fluvastatin utilization were overestimated during the entire study period, reaching an inconsistency of 15126804 doses to 27403789 DDDs in 2008.

Finally, Figure 3 shows the differences between the WHOassigned DDD (mg) with the annual average prescribed doses in Portugal. No differences were observed for cerivastatin, pitavastatin or rosuvastatin. However, the average doses of lovastatin prescribed in the past 18 years (approximately $20 \mathrm{mg}$ ) were below the assigned DDDs during the entire period, which varied from 30 to $45 \mathrm{mg}$. 
FIGURE 1 Influence of the use of different drug utilization metrics on statin consumption estimates in Portugal
Blue line: DDDYEAR/1000 inhabitants/day

Green line: PDDYEAR/1000 inhabitants/day

Red line: $\mathrm{DDD}_{\mathrm{LAST}} / 1000$ inhabitants/day

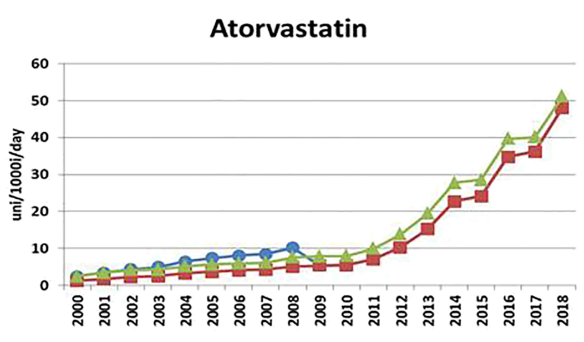

Fluvastatin
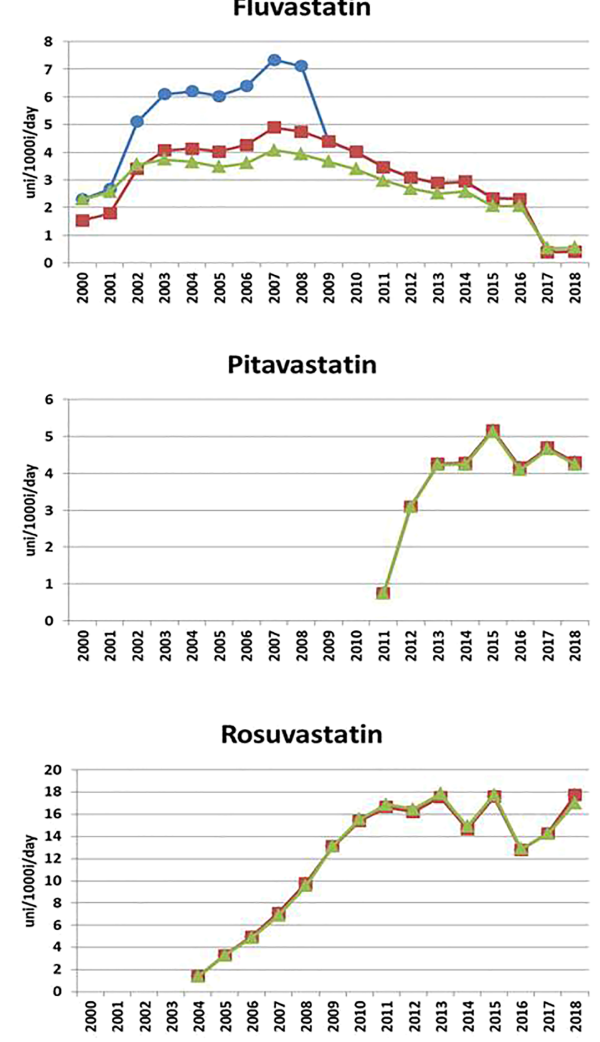

Cerivastatin

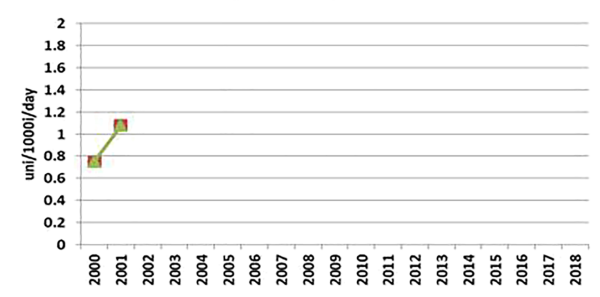

Lovastatin
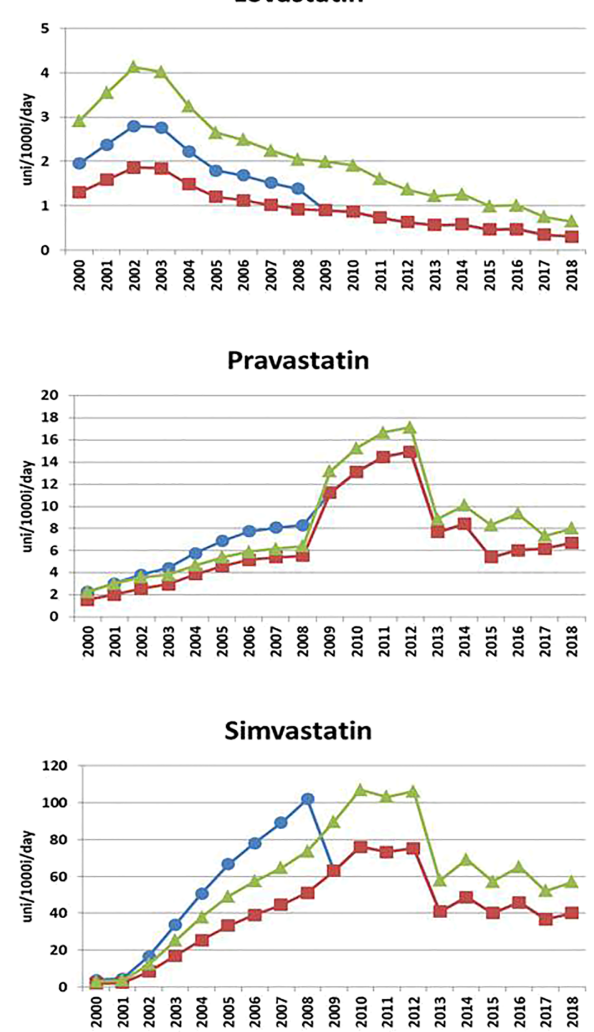

Conversely, the PDD for fluvastatin was above the DDD values during almost the entire study period (ranging from $40 \mathrm{mg}$ in 2000 to approximately $70 \mathrm{mg}$ in 2016). For atorvastatin, pravastatin and simvastatin, national PDDs were above the assigned DDD until the DDD modification in 2009.

\section{4 | DISCUSSION}

In an 18-year population-level study on statin utilization in Portugal, we were able to demonstrate several discrepancies between PDDs and DDDs, which may significantly impact the results of DUR studies in a given country or region. Therefore, when changes to the prescribed doses are not followed by analogous modifications to the DDD value assigned by the WHO-CC-DMS, the number of patients estimated to receive a medication may not correspond to the reality.
By comparing PDD $_{\text {YEAR }} / 1000$ inhabitants/day with DDD YEAR $/$ 1000 inhabitants/day, we showed that, for some statins, estimates of the fraction of drug users in Portugal were often highly under- or overestimated (i.e., values ranged between $30 \%$ and $80 \%$ ). These results were similar to those of Deambrosis et al. ${ }^{23}$ in a 10 -year study on statin utilization in Italy, reporting differences between DDD and PDD of up to $50 \%$ for some drugs, while in other cases, a perfect agreement was found between these two metrics. Another study also found different levels of discrepancies between DDDs and PDDs depending on the statin analysed in Norwegian counties. ${ }^{24}$ The authors showed that, in 2004, the DDD of lovastatin greatly corresponded to the actual mean PDD, whereas the same was not observed for the remaining statins. Conversely, our results showed that the DDD of lovastatin in 2004 performed poorly in estimating the corresponding PDD. These findings suggest that the level of concordance between DDDs and PDDs does not follow a steady pattern 
Blue line: DDD YEAR $/ 1000$ inhabitants/day

Red line: $\mathrm{DDD}_{\text {LAST }} / 1000$ inhabitants/day

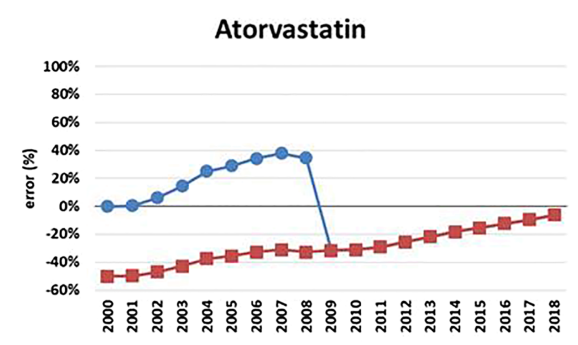

Fluvastatin
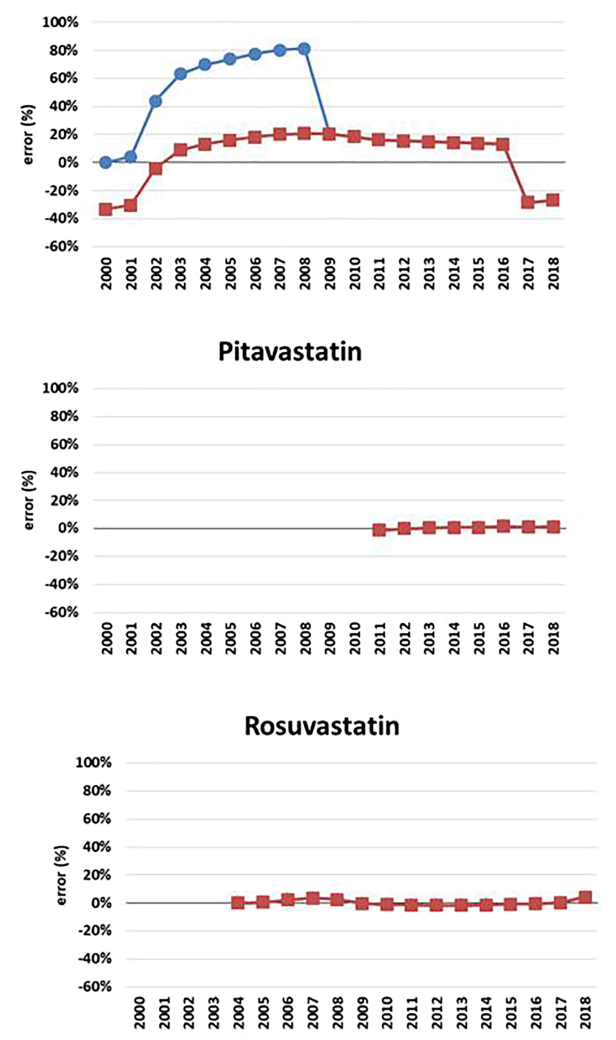

Cerivastatin

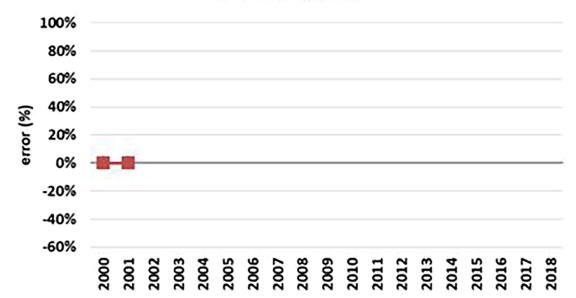

Lovastatin

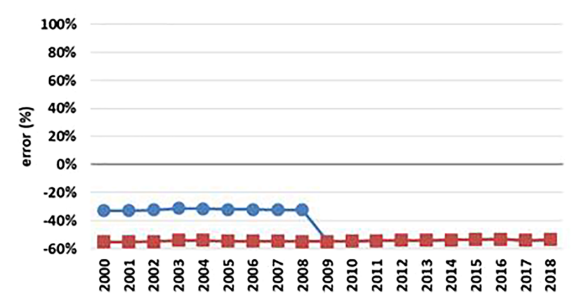

Pravastatin
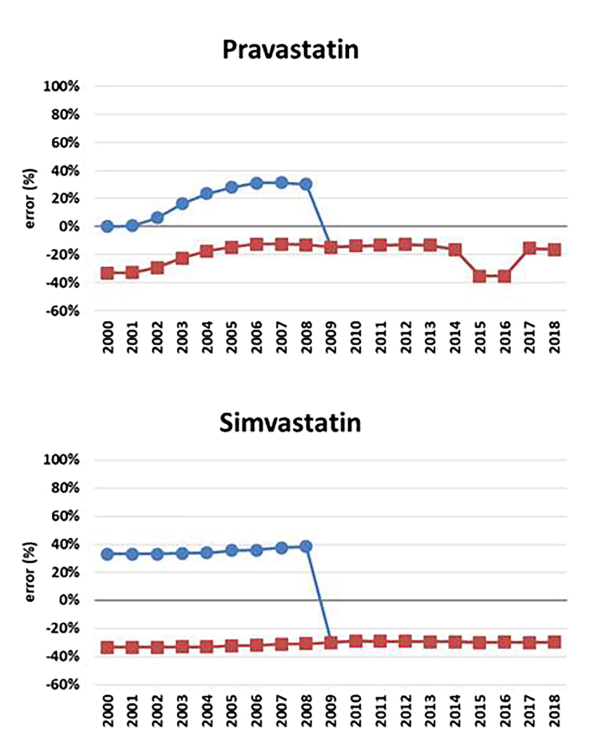

FIGURE 2 Mean percent errors of national DDD values compared with PDD and is likely to vary depending on the country, drug and year, as previously suggested by Walley et al. ${ }^{5}$ Thus, maintaining a fixed database may not be the best approach.

Both the DDD YEAR and the DDD LAST approach failed to accurately estimate the actual trend in statin utilization determined by the PDD YEAR approach. In 2009, modifications made by the WHO-CC-DSM to several statin DDDs ${ }^{10}$ resulted in abrupt and unrealistic changes in the patterns of statin utilization in Portugal, with modifications ranging from approximately $20 \%$ to $100 \%$. When DDDs are modified, the WHO-CC-DSM recommends that DUR studies use the DDD LAST approach when calculating drug utilization figures. ${ }^{12}$ However, our analyses showed that, although the DDD LAST approach may partially overcome this issue, the results of DDD LAST are not in complete agreement with PDD data. Additionally, using the DDD LAST approach, a statin utilization study published the year before DDD modifications (i.e., 2008) would have results that are not amenable to comparison with a study published at a later date.
However, although the PDDs of several statins have been modified over the years, we observed that drug utilization data calculated by the PDD YEAR approach were artifact-free. A potential explanation is that changes in PDDs occurred gradually from year to year, thus preventing abrupt modifications. As such, contrary to the WHOCC-DSM statement that "too many alterations will always be disadvantageous for long-term studies on drug utilization" and the subsequent recommendation that "changes are generally not made unless they are at least on the order of 50", ${ }^{10}$ our results suggest that more frequent changes to DDDs will be of great advantage for DUR studies.

As recognized by the WHO-CC-DSM, the purpose of expressing standardized drug utilization figures in DDD/1000 inhabitants/day is to allow benchmarking between countries so that potential misuses of drugs can be identified. ${ }^{12}$ Unfortunately, our results demonstrated that comparing crude values of DDD/1000 inhabitants/day without validating the DDD assigned by the 
FIGURE 3 Comparison of DDD assigned by $\mathrm{WHO}$ and average PDD in Portugal
Black line: $\mathrm{DDD}_{\mathrm{WHO}}(\mathrm{mg})$

Blue line: average national PDD
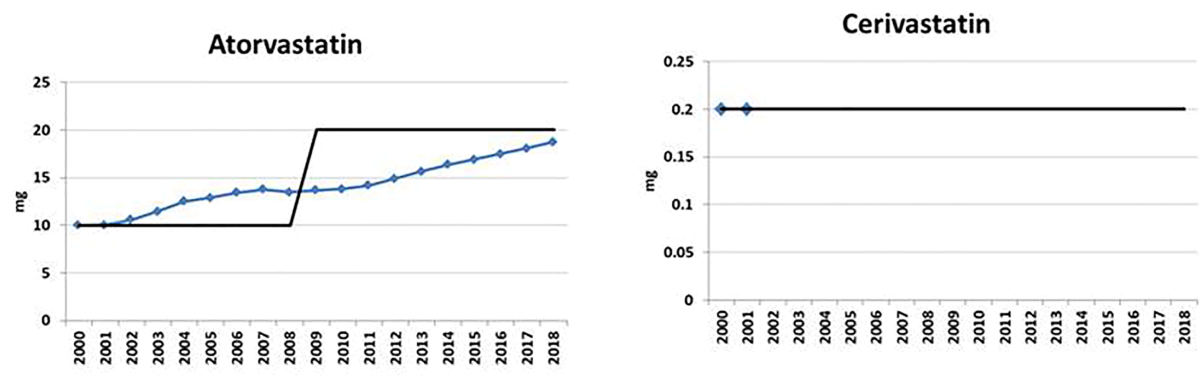

Fluvastatin
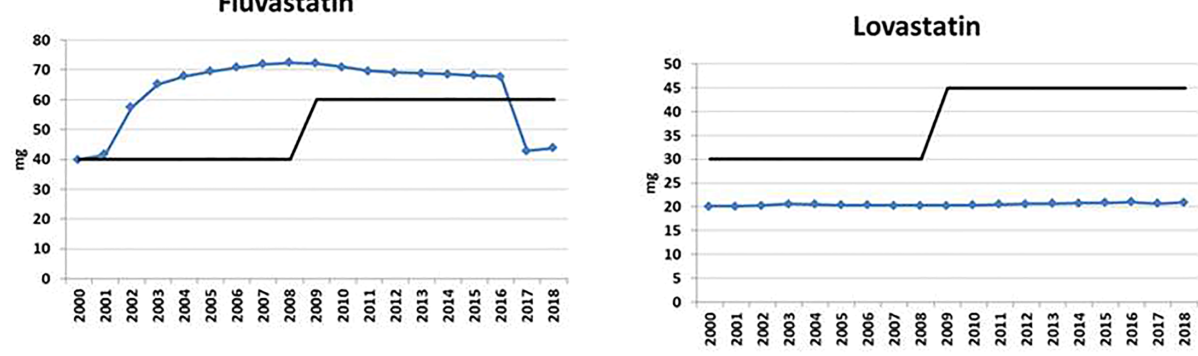

Pitavastatin

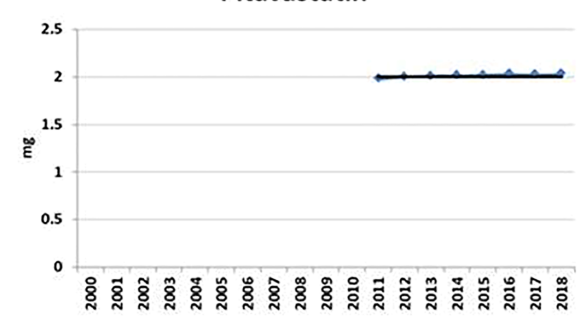

Pravastatin

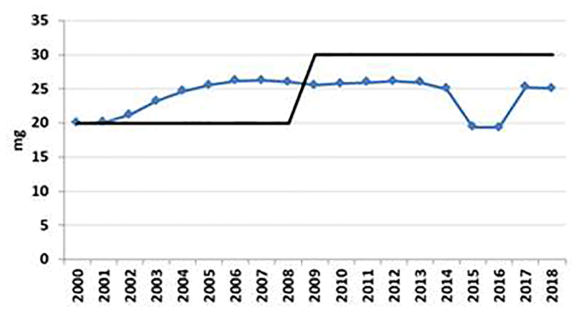

Rosuvastatin

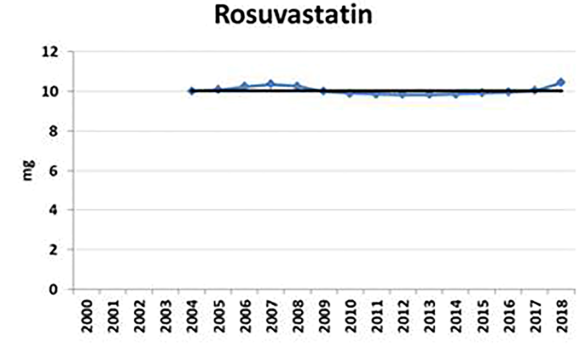

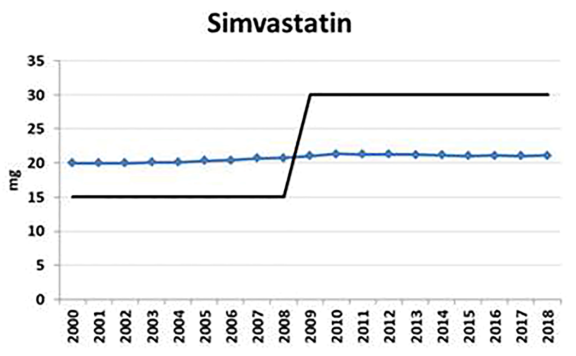

WHO-CC-DSM with local PDD studies will result in misleading comparisons and fallacious exercises.

A fixed database of DDDs brings this metric near to a "unit of mass" without any benefit over other "units of volume", such as number of packages/tablets or number of prescriptions. This steady conception of the DDD as the basis for DUR calculations weakens the DDD system and prompts researchers to develop other units of measurement as a solution to overcome discrepancies between PDDs and DDDs. ${ }^{25,26}$ Rather, we advocate for a change in the WHOCC-DSM principles for managing DDDs. Based on our results, the following alterations should be considered: first, since PDDs commonly vary between countries, when robust data about discrepancies of PDD and DDD can be demonstrated by national studies performed under the supervision of WHO-CC-DSM, national DDDs instead of international ones should be assigned to each drug; second, the
WHO-CC-DSM should encourage national regulatory bodies to periodically submit the results of prescription studies with the aim of reviewing and changing DDDs on an annual basis; third, alterations of DDDs should be made whenever changes in PDDs occur, even if the changes are small in magnitude. These considerations do not aim to suspend the WHO DDD system, but to improve inter-country comparability of drug consumption estimates. The current WHO-CC-DSM DDD database consists of a single fixed list of DDDs assigned to each drug for all the countries. We propose an integrated database where users can select the country for which DDDs are provided. When WHO-CC-DSM has no robust data to support the modification of a DDD in a given country, international DDDs will automatically be available. In this context, a strengthened DDD system could also be recommended to guide policy decisions on drug costs, pricing, reimbursement and cost containment. For example, our results 
demonstrated that a simple analysis of statin cost per patient, using the DDD/1000 inhabitants/day as a metric of statin users in Portugal, would substantially underestimate the cost of lovastatin and overestimate the cost of fluvastatin.

Our study has some strengths and limitations. Long-term longitudinal analyses of prescribing data allowed inconsistencies to be identified in the most commonly used metric in drug utilization studies. On the other hand, as we did not have access to the information on the actual PDD, this figure was determined from aggregated data based on the assumption that all patients took one tablet per day. Given the many dosages of statins available and their once-daily administration, the dispensed dose of a given statin is likely to highly agree with its actual PDD. ${ }^{21}$ Other authors have already used the same approach to determine the PDDs of statins from databases. $^{23,24}$

\section{5 | CONCLUSION}

The principle of maintaining a stable DDD system (i.e., "fixed database") should be reconsidered by the WHO-CC-DSM. A more flexible and dynamic system, based on an annually updated national DDD, would be able to reduce discrepancies between DDDs and PDDs, as well the bias in DUR studies. Further studies are needed to address other limitations of this system, such as the influence of different age pyramid profiles in DUR studies due to the different proportions of paediatric patients.

\section{ACKNOWLEDGEMENT}

This study had no external funding.

\section{COMPETING INTERESTS}

There are no competing interests to declare.

\section{CONTRIBUTORS}

The study was conceptualized by C.F., I.V.F. and F.F.-L. The data was curated by C.A. and J.R.P. Formal analysis of the data was carried out by C.A., F.S.T. and F.F.-L. The data were validated by J.R.P., M.C.-B., C.F., I.V.F. and F.F.-L. The work was supervised by C.F., I.V.F. and F.F.-L. The original draft was prepared by C.A., F.S.T., J.R.P. and F.F.-L. and all authors were responsible for reviewing and editing of the manuscript.

\section{DATA AVAILABILITY STATEMENT}

Data are available upon reasonable request.

\section{ORCID}

Fernanda S. Tonin (D) https://orcid.org/0000-0003-4262-8608 Joana Reis-Pardal (D) https://orcid.org/0000-0002-0314-8233 Margarida Castel-Branco (D) https://orcid.org/0000-0002-6533-9932 Isabel V. Figueiredo (D) https://orcid.org/0000-0003-0127-4575 Fernando Fernandez-Llimos (D) https://orcid.org/0000-0002-85299595

\section{REFERENCES}

1. Bergman U. The history of the Drug Utilization Research Group in Europe. Pharmacoepidemiol Drug Saf. 2006;15:95-98. https://doi.org/ 10.1002/pds.1171

2. Magrini N, Einarson $\mathrm{T}$, Vaccheri $\mathrm{A}$, McManus $\mathrm{P}$, Montanaro $\mathrm{N}$, Bergman U. Use of lipid-lowering drugs from 1990 to 1994: an international comparison among Australia, Finland, Italy (Emilia Romagna Region), Norway and Sweden. Eur J Clin Pharmacol. 1997;53:185-189. https://doi.org/10.1007/s002280050360

3. The EURO-MED-STAT Group. EURO-MED-STAT: monitoring expenditure and utilization of medicinal products in the European Union countries: a public health approach. Eur J Public Health. 2003;13: 95-100. https://doi.org/10.1093/eurpub/13.suppl_1.95

4. Cooke C, Nissen L, Sketris I, Tett SE. Quantifying the use of the statin antilipemic drugs: comparisons and contrasts between Nova Scotia, Canada, and Queensland, Australia. Clin Ther. 2005;27:497-508. https://doi.org/10.1016/j.clinthera.2005.04.009

5. Walley T, Folino-Gallo P, Schwabe U, Van Ganse E, Stephens P. Comparison of national administrative and commercial databases to monitor expenditure and costs of statins across Europe. Eur J Clin Pharmacol. 2004;60:503-511. https://doi.org/10.1007/s00228-0040801-9

6. Fleetcroft R, Schofield P, Ashworth M. Variations in statin prescribing for primary cardiovascular disease prevention: cross-sectional analysis. BMC Health Serv Res. 2014;14:414. https://doi.org/10.1186/ 1472-6963-14-414

7. Sabo A, Tomic Z, Stilinovic N, et al. Consumption of serum lipidreducing drugs in Serbia compared with Scandinavian countries: a population-based study, 2004-2008. Pharmacoepidemiol Drug Saf. 2011;20:45-49. https://doi.org/10.1002/pds.2045

8. Fuksa L, Vocelka M, Vytrisalova M. The impact of changes in national prescribing conditions for statins on their public expenditure and utilization in the Czech Republic 1997-2013. Health Policy. 2015;119:1255-1264. https://doi.org/10.1016/j.healthpol. 2015.02.016

9. Vancheri F, Backlund L, Strender LE, Godman B, Wettermark B. Time trends in statin utilisation and coronary mortality in Western European countries. BMJ Open. 2016;6:e010500. https://doi.org/10. 1136/bmjopen-2015-010500

10. World Health Organization Drug Utilization Research Group. https:// www.whocc.no/ddd

11. WHO International Working Group for Drug Statistics Methodology. Introduction to Drug Utilization Research. Oslo: WHO; 2003.

12. WHO Collaborating Centre for Drug Statistics Methodology. Guidelines for ATC Classification and DDD Assignment 2020. Oslo: WHO; 2019.

13. Arnold FL, Kusama M, Ono S. Exploring differences in drug doses between Japan and Western countries. Clin Pharmacol Ther. 2010;87: 714-720. https://doi.org/10.1038/clpt.2010.31

14. Nakashima K, Narukawa M, Kanazu Y, Takeuchi M. Differences between Japan and the United States in dosages of drugs recently approved in Japan. J Clin Pharmacol. 2011;51:549-560. https://doi. org/10.1177/0091270010375958

15. Malinowski HJ, Westelinck A, Sato J, Ong T. Same drug, different dosing: differences in dosing for drugs approved in the United States, Europe, and Japan. J Clin Pharmacol. 2008;48:900-908. https://doi. org/10.1177/0091270008319794

16. Grimmsmann T, Himmel W. Discrepancies between prescribed and defined daily doses: a matter of patients or drug classes? Eur J Clin Pharmacol. 2011;67:847-854. https://doi.org/10.1007/s00228-0111014-7

17. Walley T, Folino-Gallo P, Stephens P, Van Ganse E. Trends in prescribing and utilization of statins and other lipid lowering drugs across Europe 1997-2003. Br J Clin Pharmacol. 2005;60:543-551. https:// doi.org/10.1111/j.1365-2125.2005.02478.x 
18. Muller A, Monnet DL, Talon D, Henon T, Bertrand X. Discrepancies between prescribed daily doses and WHO defined daily doses of antibacterials at a university hospital. Br J Clin Pharmacol. 2006;61: 585-591. https://doi.org/10.1111/j.1365-2125.2006.02605.x

19. de With K, Bestehorn H, Steib-Bauert M, Kern WV. Comparison of defined versus recommended versus prescribed daily doses for measuring hospital antibiotic consumption. Infection. 2009;37:349-352. https://doi.org/10.1007/s15010-008-8138-4

20. Hsieh LP, Huang CY. Antiepileptic drug utilization in Taiwan: analysis of prescription using National Health Insurance database. Epilepsy Res. 2009;84:21-27. https://doi.org/10.1016/j.eplepsyres.2008. 11.018

21. Kanstrup H, Lassen JF, Heickendorff L, Lauritzen T, Larsen ML. Quality of lipid-lowering therapy in patients with ischaemic heart disease: a register-based study in 3477 patients. J Intern Med. 2004; 255:367-372. https://doi.org/10.1111/j.1365-2796.2003.01299.x

22. Vlahovic-Palcevski V, Gantumur M, Radosevic N, Palcevski G, Vander Stichele R. Coping with changes in the Defined Daily Dose in a longitudinal drug consumption database. Pharm World Sci. 2010;32: 125-129. https://doi.org/10.1007/s11096-010-9366-0

23. Deambrosis P, Saramin C, Terrazzani G, et al. Evaluation of the prescription and utilization patterns of statins in an Italian local health unit during the period 1994-2003. Eur J Clin Pharmacol. 2007;63: 197-203. https://doi.org/10.1007/s00228-006-0239-3
24. Hartz I, Sakshaug S, Furu K, et al. Aspects of statin prescribing in Norwegian counties with high, average and low statin consumptionan individual-level prescription database study. BMC Clin Pharmacol. 2007;7:14. https://doi.org/10.1186/1472-6904-7-14

25. Walley T, Roberts D. Average daily quantities: a tool for measuring prescribing volume in England. Pharmacoepidemiol Drug Saf. 2000;9: 55-58. https://doi.org/10.1002/(SICI)1099-1557(200001/02)9:1\%3 C55::AID-PDS467\%3E3.0.CO;2-H

26. Harris CM, Cullen JW, Roberts DJ. Consider national standards for daily dosages. BMJ. 1994;308:207. https://doi.org/10.1136/bmj.308. 6922.207

How to cite this article: Abrantes C, Tonin FS, Reis-Pardal J, et al. Implications of a defined daily dose fixed database for drug utilization research studies: The case of statins in Portugal. Brit Jnl Clinical Pharma. 2021;87(9):3542-3549. https://doi.org/10.1111/bcp.14770 